\title{
COMPARATIVO DE DIMENSIONAMENTO PARA EXECUÇÃO ENTRE LIGAÇÕES PARAFUSADAS POR MEIO DE PARAFUSOS DE ALTA RESISTÊNCIA E LIGAÇÕES SOLDADAS COM ELETRODO REVESTIDO
}

\section{DIMENSIONING COMPARATIVE FOR EXECUTION BETWEEN BOLTED CONNECTIONS THROUGH HIGH RESISTANCE BOLT AND WELDED CONNECTIONS WITH COATED ELECTRODE}

\author{
Tiago Aparecido de Oliveira'; Ródney Silvério Costa Junior²; Wesley \\ Cândido Silva ${ }^{3}$; André Luís Gamino ${ }^{2}$ \\ ${ }^{1}$ Universidade Paulista - UNIP, Curso de Engenharia Civil, Araçatuba, SP - Brasil \\ E-mail: tiagoengenharia52@gmail.com \\ ${ }^{2}$ Centro Universitário Católico UniSALESIANO, Curso de Engenharia Civil, Araçatuba, SP - \\ Brasil \\ E-mail: juniorcivil.sale@hotmail.com \\ ${ }^{3}$ Universidade Estadual Paulista - UNESP, Programa de Mestrado de Engenharia Civil, \\ Ilha Solteira, SP - Brasil \\ E-mail: wesley wcs@hotmail.com \\ ${ }^{4}$ Centro Universitário Católico UniSALESIANO \\ Prof. Dr. do curso de Engenharia Civil \\ Araçatuba, SP - Brasil \\ andré.gamino@gmail.com
}

RESUMO - As indústrias siderúrgicas oferecem nos dias atuais uma grande diversidade de opções para se usar o aço na construção civil, dentre elas as chapas finas, chapas grossas, parafusos, materiais para solda, barras, tubos, cabos entre outros, assim, possibilitando a construção de uma estrutura metálica. Pensando na construção da mesma, logo imaginamos como são as uniões das partes desta estrutura. Levando isso em consideração, o presente trabalho teve por escopo o dimensionamento de um galpão industrial estruturado em aço, com a finalidade de demonstrar as ligações viga-pilar e viga-viga presentes no mesmo, tratando-se de ligações com parafusos de alta resistência ASTM A325, e a ligações soldadas com eletrodo revestido. Explicitaram-se as vantagens e desvantagens dos dois processos, para demonstração da melhor ligação para o caso proposto. Realizou-se uma análise teórica dos dois casos, seguido do dimensionamento e verificações para um galpão obtendo como resultado a utilização das quantidades de parafuso de alta resistência A325 para a seção rotulada e dimensão e tipo de solda para seção engastada.

Palavras-chave: Aço ASTM A572. Dimensionamento. Estrutura metálica. Ligações parafusadas. Ligações soldadas.

Recebido em: $17 / 08 / 2018$ Revisado em: 09/09/2018 Aprovado em: 18/09/2018
ABSTRACT - The steel industry offers a wide variety of options for using steel in construction, such as thin steel, sheet metal, screws, welding materials, bars, cables, cables among others, thus making it possible to build a metal structure. Thinking about the construction of the same, 
then we imagine as the unions of the parts of the structure. Taking this into consideration, the present work has as scope the design of an industrial shed structured in steel, with the purpose of demonstrating how the beams-abutments and beam-beams connections do not even present, when screwing with high resistance screws ASTM A325, and welded connections with coated electrode. The advantages and disadvantages of the two processes were explained for a better connection to the proposed case. A theoretical analysis of the two cases was carried out, followed by sizing and checking to obtain an indication of the type of A325 high strength screw support for a section labeled and size and type of weld for the crimped section.

Keywords: Metallic Structure. Bolted Connections. Welded Connections. Steel ASTM A572. Dimensioning.. 


\section{INTRODUÇÃO}

O aço atualmente pode ser existente na construção civil tanto como material principal, como parte das obras, considerando que irá construir uma estrutura de aço, deve-se ser pensado em como será feito a união desse material, já que se tratam de ser peças vindas de fábrica (FERRAZ, 2003).

A expressão ligação tem por significado a união de duas ou mais partes de um determinado corpo, tornando-as assim em um único elemento. É primordial o uso de ligações nas estruturas metálicas para unir partes constituintes na estrutura, podendo ser classificadas em ligações permanentes e ligações desmontáveis. As permanentes são as que tornam as peças em apenas uma, enquanto as desmontáveis são as que possuem um dispositivo entre as peças. Neste trabalho optou-se por ligações permanentes as soldadas com eletrodo revestido (SMAW) e para as ligações desmontáveis, as parafusadas com parafusos de alta resistência (ASTM A325) (VASCONCELOS, 2014).

As ligações parafusadas proporcionam uma ligação indireta das peças, ou seja, entre elas existem dispositivos, sendo eles chapas e os próprios parafusos, e são empregados geralmente em estruturas destinadas a receber grandes cargas nas peças (GOECKS, 2011). Os parafusos de alta resistência ASTM A325 (figura 1) são os mais utilizados devido a sua resistência ser maior que os outros existentes (BELLEI, 2008).

A soldagem é um processo que proporciona a junção direta de duas ou mais partes na estrutura, dando assim uma continuidade perfeita (VASCONCELOS, 2014). A solda com eletrodo revestido (figura 2) é a mais utilizada em indústrias, podendo ser aplicada tanto em instalações industriais consideradas pesadas, quanto em pequenos trabalhos em campo, exigindo maior tempo e cuidado em sua montagem (BELLEI, 2008).

Figura 1. Ligações parafusadas com parafusos de alta resistência

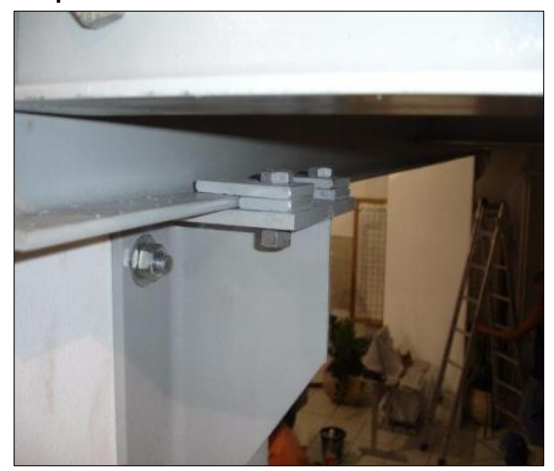

Fonte: Próprio Autor

Assim, pretende-se neste trabalho o dimensionamento de um galpão industrial estruturado em aço, com a finalidade de demonstrar as ligações vigapilar e viga-viga presentes no mesmo, tratando -se de ligações com parafusos de alta resistência ASTM A325, e a ligações soldadas com eletrodo revestido. 
Figura 2. Ligações parafusadas com eletrodo revestido

Fonte: Próprio Autor

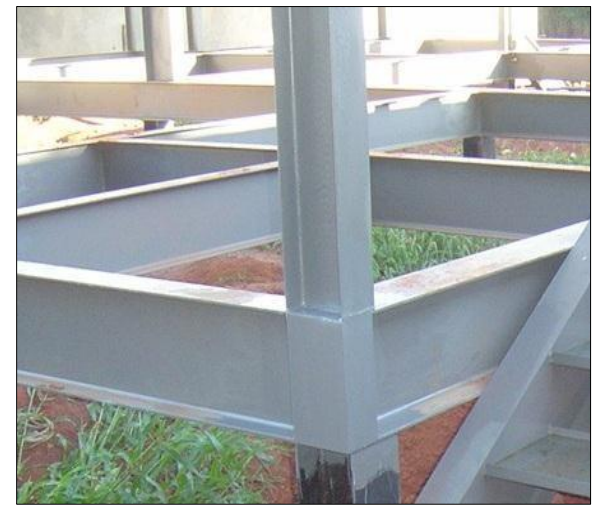

\subsection{AÇO ASTM A572}

$O$ aço pode ser categorizado como aço carbono de media resistência, aço de alta resistência e baixa liga, e aço tratado termicamente. O aço ASTM A572 está na categoria de alta resistência e baixa liga, analisado na tabela 1 (LERIS, 2010). As estruturas metálicas requerem um bom

aço que atenda as seguintes características: boa ductilidade, homogeneidade e soldabilidade, o aço ASTM A572 carrega essas características, sendo ele o mais utilizado na construção civil (PFEIL, 2009).

Tabela 1: Classificação do Aço.

Fonte: Catalogo Aço na construção civil CNS.

\begin{tabular}{|c|c|}
\hline Especificação & $\begin{array}{c}\text { ASTM } \\
\text { A572 }\end{array}$ \\
\hline Granulometria & 50 \\
\hline Teor de Carbono & 0,23 \\
\hline $\begin{array}{c}\text { Limite de Escoamento fy } \\
\text { (Mpa) }\end{array}$ & 345 \\
\hline Resistência a Ruptura (Mpa) & 450 \\
\hline
\end{tabular}

\subsection{ELEMENTOS DE LIGAÇÃO: LIGAÇÃO PARAFUSADAS COM PARAFUSOS DE ALTA RESISTÊNCIA (ASTM A325)}

A fabricação dos parafusos de alta resistência (figura 4) é feita com açocarbono médio temperado, que o possibilita apresentar uma resistência de tração e cisalhamento superior aos demais existentes (GOECKS, 2011). Os mesmos são aplicados em situações que estão prestes a receber grandes cargas em suas peças a serem ligadas, e nas principais partes da estrutura submetidas a receberem cargas dinâmicas.

Os parafusos possuem porcas, que assim quando apertadas geram uma alta tensão de tração tão intensa que pelo atrito acabam impossibilitando o movimento das chapas (BELLEI, 2008).

O uso das arruelas nos parafusos é de grande importância, em razão de que elas que são as responsáveis por impedir a perda de pré-carga após o aperto (TECÉM, 2015). Os mesmos são montados com protensão, ou seja, as partes ficam pressionadas uma com a outra, por meio de cone de pressão, podendo ser também apontado como um cilindro de pressão (CEDAC, 2001). Os parafusos são alojados em chapas, que devem ser furadas, e as regras dessa furação são especificadas pela NBR 8800 (2008), proporcionando assim facilidade na fabricação e padronização (figura 5). 
Figura 4. Parafuso de alta resistência.

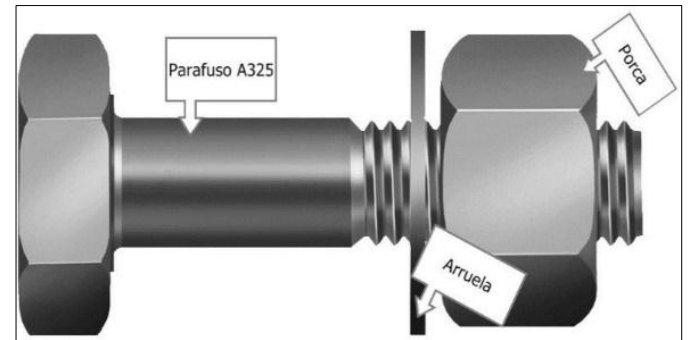

Fonte: (Disponível em: < https://bit.ly/2wdrrwn>. Acesso em: 23 ago. 2016.

Figura 5. Tipos de furos nas chapas: (a) furo padrão; (b) furo alargado; (c) furo pouco alongado; (d) furo muito alongado.

Fonte: PFEIL (2009).

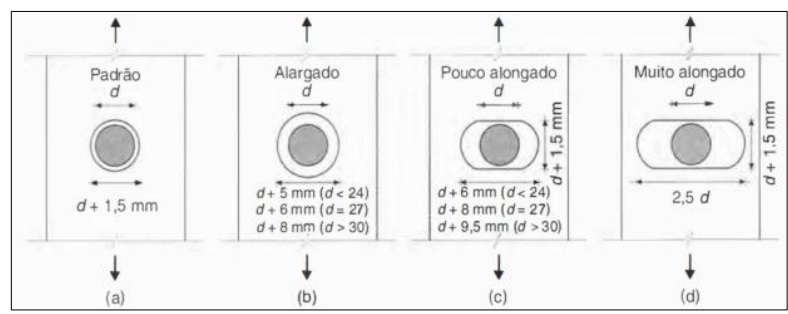

O mais usual é o tipo de furo padrão tendo seu diâmetro exatamente igual ao do parafuso somado a $1,5 \mathrm{~mm}$, os demais furos só serão utilização em casos específicos por demanda de um responsável. Os espaçamentos são esclarecidos pela NBR 8800:2008, e os resultados estão mostrados na tabela 3 .

Tabela 2:. Piores tensões ocorrentes no pórtico

\begin{tabular}{|c|c|c|c|c|c|c|}
\hline \multicolumn{7}{|c|}{ Verificação de Resistência } \\
\hline \multirow{3}{*}{ Barra } & \multicolumn{7}{|c|}{ Esforços desfavoráveis } \\
\cline { 2 - 7 } & $\begin{array}{c}\mathrm{N} \\
(\mathrm{t})\end{array}$ & $\begin{array}{c}\mathrm{Vy} \\
(\mathrm{t})\end{array}$ & $\begin{array}{c}\mathrm{Vz} \\
(\mathrm{t})\end{array}$ & $\begin{array}{c}\mathrm{Mt} \\
(\mathrm{t} \cdot \mathrm{m})\end{array}$ & $\begin{array}{c}\mathrm{My} \\
(\mathrm{t} \cdot \mathrm{m})\end{array}$ & $\begin{array}{c}\mathrm{Mz} \\
(\mathrm{t} \cdot \mathrm{m})\end{array}$ \\
\hline $\mathrm{N} 1 / \mathrm{N} 2$ & 1.689 & 0.000 & -0.401 & 0.000 & 5.188 & 0.000 \\
\hline $\mathrm{N} 2 / \mathrm{N} 3$ & 0.783 & 0.000 & 1.871 & 0.000 & 5.188 & 0.000 \\
\hline $\mathrm{N} 4 / \mathrm{N} 3$ & -0.980 & 0.000 & -1.456 & 0.000 & -3.376 & 0.000 \\
\hline $\mathrm{N} 5 / \mathrm{N} 4$ & -2.070 & 0.000 & -0.675 & 0.000 & 3.376 & 0.000 \\
\hline
\end{tabular}

Fonte: Relatório do Software Computacional Metálica 3D

\section{METODOLOGIA}

Para o prosseguimento do trabalho, e para que fosse alcançado os objetivos citados, desenvolveu-se primeiramente a fundamentação teórica do assunto tratado, para que logo após fosse possível ser executado o dimensionamento de um galpão para depósito no Distrito Industrial de Araçatuba, com baixo fator de ocupação. O mesmo possui as seguintes especificações: Cobertura e fechamento nas 4 faces com telhas em aço galvanizado tipo trapezoidal, executada em um terreno plano, com dimensões do galpão $10 \times 40 \mathrm{~m}$, sendo um Pórtico em perfis $W$ com aço ASTM A572 - Grau 50.

Baseou-se os cálculos na NBR 8800:2008 - Projeto de estruturas de aço e de estruturas mistas de aço e concreto de edifícios. Para as solicitações de cálculo, utilizou-se as piores tensões atuantes na estrutura (figura 3), calculados a partir do software Metálica 3D, para dimensionamento das colunas e vigas tem-se as tabelas 2 a seguir: 
Figura 3. Modelo proposto para cálculo. Croqui representativo, sem escala. Software Metálica 3D.

Fonte: Próprio Autor

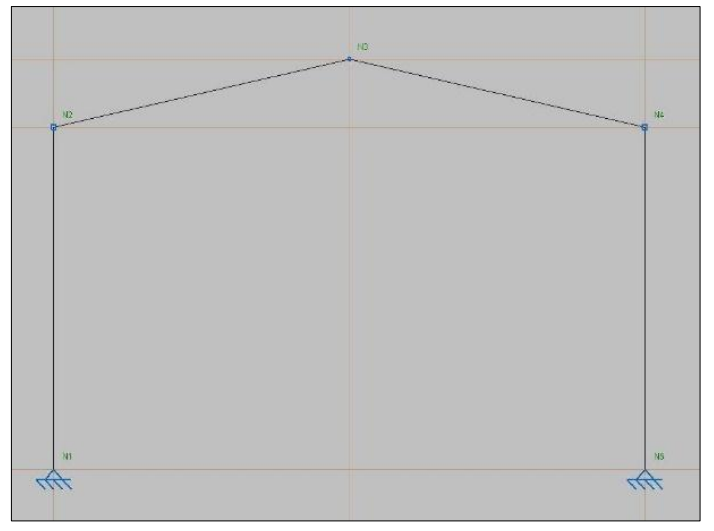

O processo de solda consiste na ligação direta dos elementos, sendo necessário que haja uma energia aplicada sobre os elementos para que ocorra a fusão, podendo ser energia elétrica, química, óptica ou mecânica. A energia aplicada em construções civis é a energia elétrica, a mais comum, posiciona-se o eletrodo o mais próximo das partes a serem soldadas, formando um arco voltaico que acaba sendo responsável pela liberação de calor, causando a fusão do aço, o material que se funde deve ser isolado da atmosfera para que não haja presença de impurezas, que possam causar danos na solda, nesse caso será usado a proteção por eletrodo revestido. A finalidade na prática da soldagem é que se obtenha uma solda de boa qualidade, que não apresente fraturas e que seja resistente (PFEIL, 2009).

Alguns cuidados específicos devem ser analisados no momento em que for realizar a solda, pois se não os precaver podem ocorrer alguns defeitos catastróficos e irreparáveis na estrutura, como por exemplo fraturas a frio ou a quente, fusão incompleta, porosidade, inclusão de escória, conforme é exemplificada na figura 6 , e as soldas apresentam diversos tipos de cordões, sendo eles: solda de filete, solda de penetração total e solda de penetração parcial conforme explicita a figura 7 :

Figura 6. Defeitos na solda

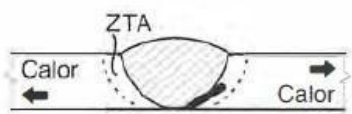

(a) Fraturas a frio

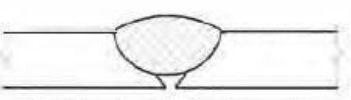

(c) Penetração inadequada

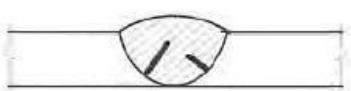

(b) Fraturas a quente

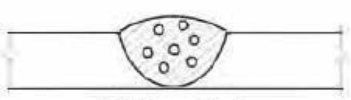

(d) Porosidade

Fonte: (PFEIL, 2009). 
Figura 7. Cordões da solda.

Fonte: (PFEIL, 2009).

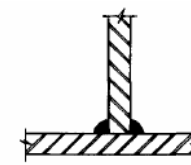

(a) SOLDA DE FILETE

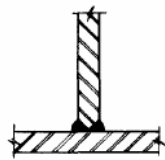

(b) SOLDA DE PENETRACĀO
TOT A L.

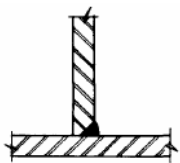

(c) SOLDA DE PENETRACĀO
PARCIAL.
Escolheu-se o cordão de solda de filete, por ser o tipo mais utilizado, e o mais simples de se executar, poderia trazer tudo, conforme será exemplificado no artigo para (VALENCIANI, 1997), a solda de filete possui as seguintes características:
Raiz da solda; Face de fusão; Perna do filete; Garganta efetiva; Comprimento efetivo; Área efetiva, conforme figura 8 (PFEIL, 2009):

Figura 8. Características da solda filete
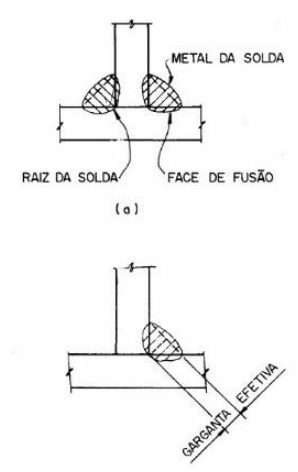

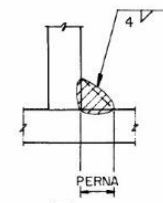

(b)
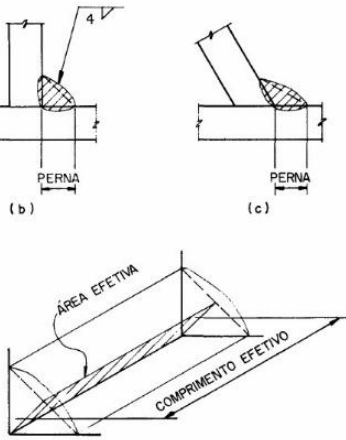

(c)

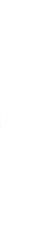

Fonte: Ligações em estruturas Metálicas-Manual Centro Brasileiro da Construção em Aço(CBCA).

\section{RESULTADOS}

Abaixo, os resultados obtidos utilizando o software Metálica 3D:

Tabela 3. Resultados das forças resistentes obtidas para o parafuso ASTM A325

\begin{tabular}{|c|c|c|c|c|c|}
\hline Esforço & $\boldsymbol{F v}, \boldsymbol{s \boldsymbol { d }}$ & $\boldsymbol{F v}, \boldsymbol{R \boldsymbol { d }}$ & $\boldsymbol{F \boldsymbol { c } , \boldsymbol { R } \boldsymbol { d }}$ & $\boldsymbol{F r}, \boldsymbol{R \boldsymbol { d }}$ & Qtd \\
\hline $\begin{array}{c}\text { Seção Engastada } \\
\text { (viga-pilar) }\end{array}$ & $29,35 \mathrm{kN}$ & $61,42 \mathrm{KN}$ & $104,5 \mathrm{kN}$ & $4166,43 \mathrm{kN}$ & $\begin{array}{c}2 \mathrm{de} 16 \\
\mathrm{~mm}\end{array}$ \\
\hline $\begin{array}{c}\text { Seção rotulada } \\
\text { (viga-viga) }\end{array}$ & $12,91 \mathrm{kN}$ & $61,42 \mathrm{kN}$ & $104,5 \mathrm{kN}$ & $2149,77 \mathrm{kN}$ & $\begin{array}{c}2 \mathrm{de} 16 \\
\mathrm{~mm}\end{array}$ \\
\hline
\end{tabular}


Tabela 4. Resultados das forças resistentes obtidas para solda tipo filete

\begin{tabular}{|c|c|c|c|c|c|c|}
\hline \multicolumn{7}{|c|}{ SOLDA TIPO FILETE } \\
\hline Esforço & $\boldsymbol{S d}$ & $\boldsymbol{A w}$ & $\boldsymbol{b} \boldsymbol{m i ́ n}$ & $\boldsymbol{b}$ má $\boldsymbol{t}$ & t & $\boldsymbol{l}$ \\
\hline $\begin{array}{c}\text { Seção Engaste } \\
\text { (viga-pilar) }\end{array}$ & $27,08 \mathrm{kN}$ & $1,47 \mathrm{~cm}^{2}$ & $3 \mathrm{~mm}$ & $4,75 \mathrm{~mm}$ & $2,10 \mathrm{~mm}$ & $7 \mathrm{~cm}$ \\
\hline $\begin{array}{c}\text { Seção rotulada } \\
\text { (viga-viga) }\end{array}$ & $17,93 \mathrm{kN}$ & $0,97 \mathrm{kN}$ & $3 \mathrm{~mm}$ & $4,75 \mathrm{~mm}$ & $2,10 \mathrm{~mm}$ & $4,62 \mathrm{~cm}$ \\
\hline
\end{tabular}

\section{DISCUSSÃO}

Com a utilização do parafuso de alta resistência como conector das duas seções viga-pilar e viga-viga, teoricamente, se teria uma execução prática por ter a quantidade de material pré-estabelecido a partir dos cálculos, visto que, para este tipo de execução a fabricação das peças são rápidas, a mão de obra não necessita especialidade e não é necessária energia para montagem da mesma.

\section{CONSIDERAÇÕES FINAIS}

Com os resultados obtidos (tabela 3 e 4), considera-se que em relação ao perfil considerado, o software computacional apresentou o que melhor atendeu às cargas atuantes após verificações de resistências realizadas. Assim, foram considerados como parâmetros de comparação, estudos relacionados a forças atuantes e forças resistentes de cada método verificado.

Para seção engastada viga-pilar, perante o dimensionamento, apresentou a viabilidade da utilização dos parafusos de alta resistência. Cada seção engastada, cabe a realização de dois furos de 1,75 cm cada, resultando em $7 \mathrm{~cm}$ furado. Contudo, para execução da solda, orientase $7 \mathrm{~cm}$ em cada seção, ou seja, $14 \mathrm{~cm}$ soldados para as duas seções. Assim, com a utilização do parafuso de alta resistência $A 325$, tem-se a os elementos viga-pilar ligados através do conector de maior resistência, dando estabilidade para estrutura.

Já para seção rotulada viga-viga, comparando os dois conectores, observase que para cada seção cabe a utilização de um furo de $1,75 \mathrm{~cm}$ cada, resultando em 3,50 cm furados, e em contrapartida 4,62 cm de solda. Observa-se também que, a resistência do parafuso perante às forças atuantes, são maiores quando comparadas com a solda. Com isso se torna viável, segundo dimensionamento, a utilização do parafuso de alta resistência A325 para a seção rotulada.

\section{REFERÊNCIAS}

ALMEIDA, P. H.V. Estudo e verificação de ligações metálicas parafusadas e soldadas. 2014. 48f. Trabalho de Conclusão de Curso (Bacharelado em Engenharia Civil) Faculdades de Tecnologia e Ciências Sociais Aplicadas-FATECS, Brasília, 2014.

APOSTILA Estrutura Metálicas - Curso Profissionalizante e Supletivo Modulo II. CEDAC,2001.

ARRUELAS-Informações Gerais - Tecnologia Empresarial - TECEM. Jan 2015. Disponível em: http://www.tecem.com.br/tabelaengenharia/arruelas- informacoes-gerais/ Acesso em: 20 ago. 2018.

\section{ASSOCIAÇÃO BRASILEIRA DE NORMAS} TÉCNICAS. ABNT. NBR 8800:2008 -Projeto de estruturas de aço e de estruturas mistas de aço e concreto de edifícios. Rio de Janeiro: ABNT, 2008. 
BELLEI, I. H.; PINHO, F. O.; PINHO, M. O.

Edifícios de múltiplos andares em aço. 2.

ed. São Paulo: Pini, 2008.

GOECK, C. Análise de ligações parafusadas

em estruturas metálicas.2011. 67f.

Trabalho de Conclusão de Curso

(Bacharelado em Engenharia Civil) -

Universidade Regional do Noroeste do

Estado do Rio Grande do Sul, Itajuí, 2011.

PFEIL, W.; PFEIL, M.. Estruturas de aço:

dimensionamento prático. 8. ed. São Paulo:

LTC, 2009.

VALENCIANI, V. C.; Ligações em estruturas de aço. 1997. 252f. Dissertação (Mestrado em Engenharia Civil) - Universidade de São

Paulo, São Carlos, 1997. 